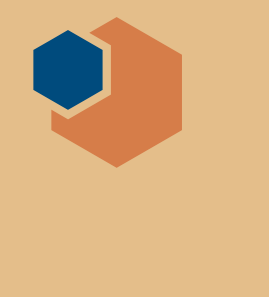

\section{US Department of Energy celebrates 10 years at the frontiers of energy research}

\author{
science.osti.gov/bes/efrc
}

$\mathrm{T}_{\mathrm{c}}^{\mathrm{n}}$ he US Energy Frontier Research Center (EFRC) Program turned 10 in 2019, and in an article to mark the milestone, Director of the US Department of Energy (DOE) Office of Science Chris Fall writes that "this challenge is a scientific one, but it's also a management one How do you bring together, empower, and support the creative, multidisciplinary, and multi-institutional scientific teams needed to tackle the toughest scientific challenges preventing advances in energy technologies?" Unsurprisingly, Fall's answer is through the EFRC program, which seeks to transform the energy landscape by both building effective teams and ensuring these teams have access to a breadth of tools to better understand and control energy-relevant materials.

The Office of Basic Energy Science within DOE launched the EFRC program in 2009 in response to the accelerating global demand for energy. To meet the energy needs of the future, researchers are working to find new ways to collect and generate energy, develop better means involve substantial materials research. energy challenges of the 21 st century. to store it, and advance technologies that increase energy efficiency, all of which

The 10-year anniversary of the EFRC program provides an opportunity to look back at the successes of the program, and look toward its future. In conjunction with the anniversary, DOE has announced winners of its video contest about the science, innovations, and people of the EFRCs as well as the "Ten at Ten Awards," which recognizes people, scientific ideas, and tools and technologies that exemplify the impact of the EFRCs (see Table I). Several of the award recipients are materials researchers and engineers - a testament to the importance of materials in solving the

Fall writes that the EFRCs were "an experiment in the management of basic research that has since paid huge dividends ..." -a statement that is backed by the numbers. In just a decade, the EFRC program has awarded nearly USD $\$ 1.6$ billion in funding to support 82 centers, 46 of which are currently active. These centers

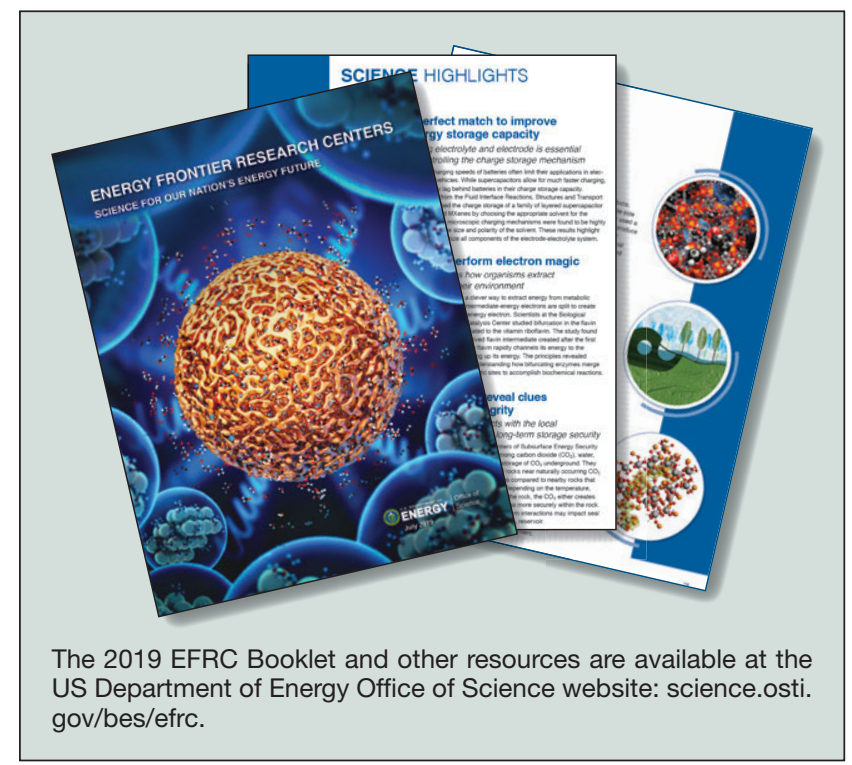
have focused on the range of energy challenges and have together produced over 11,600 peer-reviewed publications as well as a variety of novel ideas and technologies (including $650+$ invention disclosures, $550+$ US patent applications, $400+$ nonUS patent applications, and $180+$ issued patents as of 2019).
In addition to publications and the generation of intellectual property, the EFRCs have engaged scientists and researchers from more than 170 institutions. With a focus on both utilizing expertise and building the future energyscience workforce, supported researchers have hailed from a number of fields and career levels, including over 1600 senior investigators and more than 5400 students or postdoctoral researchers.

"The most significant impact of the EFRC program is the large number of talented young scientists and engineers who have been trained in interdisciplinary energy research," says Mark C. Hersam, Walter P. Murphy Professor of Materials Science and Engineering at Northwestern University, who received one of the "Ten at Ten" awards.

Erin L. Ratcliff, assistant professor of materials science and engineering at the University of Arizona and another award recipient of the "Ten at Ten," echoes Hersam's statement and expands on this, saying that she believes "centers offer a successful alternative to graduate and postdoc training methodology over conventional academic approaches - one group with one PI" because as a young researcher involved in an EFRC, you have access to "a collection of perspectives and mentorship opportunities."

The EFRCs are also "supporting young faculty as they are building up their nascent research programs," says Mercouri Kanatzidis, the Charles E. and Emma H. Morrison Professor of Chemistry at Northwestern University. This investment in people is important, according to Kanatzidis, because "in a few more years, there will be a sizable number of 'alumni' with research training and attitudes that will positively impact in solving future challenges." Hersam adds, "I anticipate that the development of the next generation of scientists and engineers will continue to be the largest impact of the EFRC program over the next decade." Kanatzidis also received the "Ten at Ten Award."

According to Ratcliff, another significant advantage of conducting research within an EFRC is access to a diverse network and the "commitment to collaboration." Ratcliff emphasizes the advantages 
of the centers' multidisciplinary teams saying that in her research, the different ways team members thought about and approached a problem, as well as the multitude of tools available across the team, led to enhanced understanding of the interfacial behaviors of solar materials.

The EFRC structure not only brings together multidisciplinary research groups, it also establishes partnerships between universities, national laboratories, and industry partners. This structure has increased the sharing of ideas, provided greater access to expertise and tools, and spanned some of the barriers between basic research and commercialization of energy technologies. At the 10-year mark, about 110 companies (ranging from startups to large corporations) have collaborated with, and benefited from, the EFRCs.

Hersam is part of a group that developed and commercialized (through the formation of a startup company) new graphene-encapsulated lithium-ion battery cathode materials - a breakthrough that he credits to the "highly collaborative" environment of the EFRCs. According to Hersam, through the EFRC "we were not only able to pursue fundamental science but also capitalize on opportunities for impacting applied technologies." Kanatzidis has had a similar experience, and says that the EFRC structure gave him and his colleagues access to tools, facilities, and talented junior researchers that allowed them to pursue and successfully demonstrate in 2012 the first-known all-solid-state solar cells using halide perovskite materials.

Another example of the value of the EFRC structure comes from Tonio Buonassisi, professor of mechanical engineering at the Massachusetts Institute of Technology, and a co-author of one of the 10 most highly cited EFRC articles. The article in MRS Communications, "Identifying defect-tolerant semiconductors with high minority-carrier lifetimes: Beyond hybrid lead halide perovskites" (doi:10.1557/mrc.2015.26), details a method that could be used more broadly to identify defect-tolerant semiconductors for a range of uses. Several of the materials identified by the proposed method have since been tested experimentally and, according to Buonassisi, they have

\section{Table I. EFRC Ten at Ten Awards.}

\section{PEOPLE AWARDS}

Erin L. Ratcliff

Center for Interface Science:

Solar Electric Materials (CISSEM)

\section{Michael Naguib}

Fluid Interface Reactions, Structures

and Transport Center (FIRST)

Katlyn M. Turner

Materials Science of Actinides

(MSA)
For embracing multidisciplinary team science to characterize and control the chemical and physical interactions between electrical contacts and active layers in emerging solar-energy technologies.

For developing a new family of two-dimensional materials based on transition-metal carbides and nitrides that have proven to be transformative battery and supercapacitor electrode materials.

For her dedication to EFRC research from undergraduate through graduate studies, transferring research knowledge and techniques between institutions, and maintaining strong ties between collaborating groups.

\section{SCIENTIFIC IDEAS AWARDS}

Mercouri Kanatzidis and Robert P.H. Chang

Argonne Northwestern Solar Energy Research (ANSER)/

Center for Light Energy Activated Redox Processes (LEAP)

Jeffrey R. Long, Thomas M. McDonald, Douglas A. Reed, Rebecca L. Siegelman, and C. Michael McGuirk

Center for Gas Separations (CGS)

Candace Haigler, James Kubicki, B. Tracy Nixon,

Hugh O'Neill, Alison Roberts, Ming Tien,

Yaroslava Yingling, and Jochen Zimmer

Center for Lignocellulose Structure and Formation (CLSF)

Carrie Siu, Yuhchieh Lin, leuan Seymor, and Jatin Rana NorthEast Center for Chemical Energy Storage (NECCES)
For the first demonstration of all-solid-state solar cells using halide perovskite materials.

For the discovery of cooperative adsorption in metal-organic frameworks.

For elucidating the structure and function of plant cellulose synthase and cellulose synthesis complex.

For developing a fully rechargeable multi-electron 2-lithium battery cathode.

\section{TECHNOLOGIES AND TOOLS AWARDS}

\section{Paul Dauenhauer}

Catalysis Center for Energy Innovation (CCEI)

Harold Kung, Cary Hayner, and Mark Hersam Center for Electrochemical Energy Science (CEES)

\section{David Bierman}

Solid State Solar Thermal Energy Conversion Center (S $\left.{ }^{3} T E C\right)$
For developing the quantitative carbon detector 3D printed metal catalytic microreactor.

For developing graphene encapsulation of lithium-ion battery anodes and cathodes.

For developing an ultrahigh efficiency thermophotovoltaic power-conversion device. been shown "to exhibit very good carriertransport properties - evidence that our approach has merit." Buonassisi explains, "In essence, we faced a tough problem that theory couldn't solve directly, so we developed proxies that got us close." But getting there required a team-one that Buonassisi says the "EFRC brought together" and included database curators, first-principle theorists, and experimentalists.

Buonassisi points out that to accelerate the rate of novel materials develop- ment (which will be required to solve many energy challenges), a combination of high-throughput experimentation, high-performance computing, and data science methods will be required. The continuing EFRC program has already begun to put the pieces and people together-building and enabling the teams of scientists and engineers that will continue to define the energy landscape of the future.

Jennifer A. Nekuda Malik 